\title{
Investigación en Educación: Manual de Prácticas para el alumno
}

Máster en Intervención en Convivencia Escolar

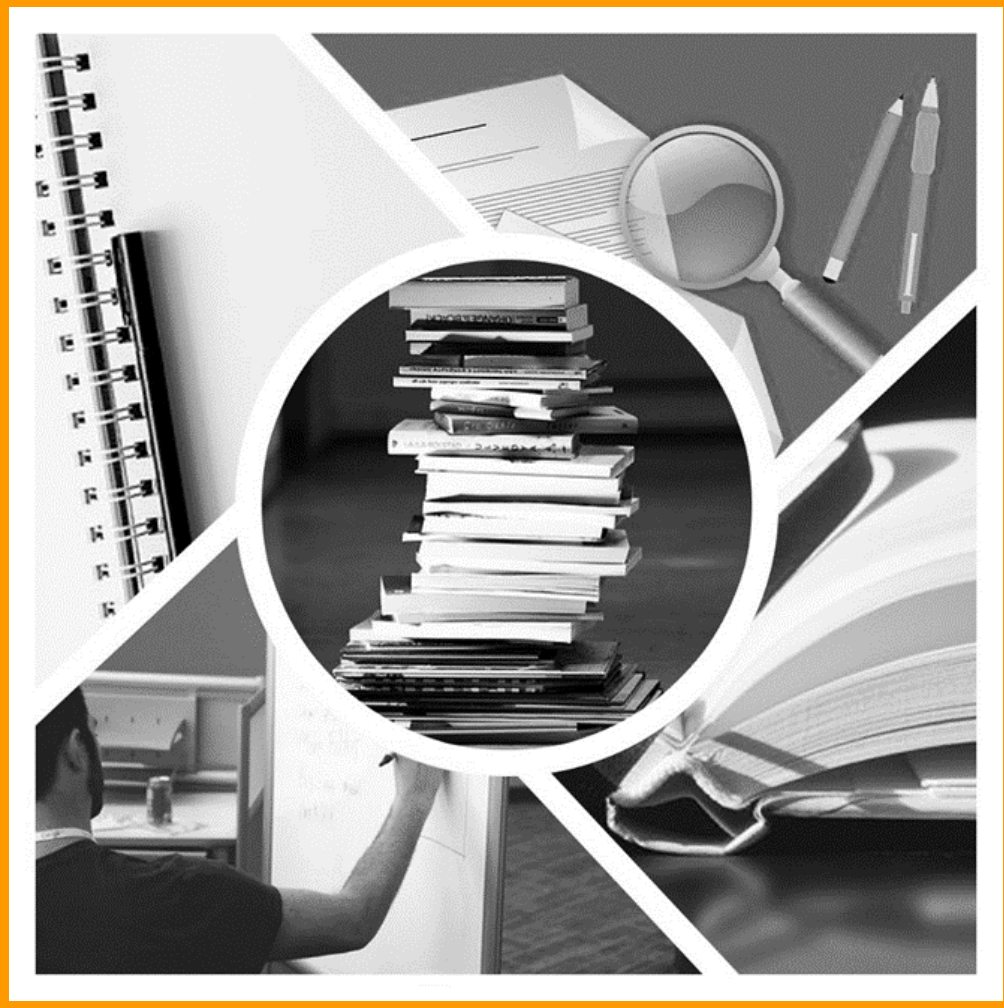

María del Carmen Pérez Fuentes María del Mar Molero Jurado África Martos Martínez María del Mar Simón Márquez Ana Belén Barragán Martín José Jesús Gázquez Linares Nieves Fátima Oropesa Ruiz 




\title{
Investigación en Educación: Manual de Prácticas para el Alumno
}

\author{
María del Carmen Pérez Fuentes \\ María del Mar Molero Jurado \\ África Martos Martínez \\ María del Mar Simón Márquez \\ Ana Belén Barragán Martín \\ José Jesús Gázquez Linares \\ Nieves Fátima Oropesa Ruiz
}


(C) Investigación en Educación: Manual de Prácticas para el Alumno Edita: Asunivep Lugar: Almería ISBN: 978-84-09-17496-6

Depósito Legal: AL 2991-2019

Impresión: ALPE GRÁFICAS DE ALMERÍA S.L.

Primera edición en Chile Universidad Autónoma de Chile ISBN (digital): 978-956-8454-84-5 
Actividades de la Asignatura de Modelos Teóricos y Bases Conceptuales en Convivencia Escolar. . .9

La Convivencia Escolar en el siglo XX............................................................ 13

Comienza a conocer la Convivencia Escolar ................................................ 15

La Convivencia Escolar en la actualidad .......................................................... 17

Comparación crítica y reflexiva.................................................................... 19

Variables y dimensiones de la convivencia escolar........................................ 21

Teorías fundamentales sobre el origen de la agresión .................................... 23

Programa de Intervención ............................................................................. 25

La resolución de conflictos en el aula............................................................... 27

Actividades de la Asignatura de Legislación Vigente en Educación y Convivencia Escolar...............................................................................................29

El origen de la educación en España y la evolución de las leyes de educación 33

Programas y organismos en Convivencia Escolar ........................................ 35

Recursos acerca de la Convivencia Escolar y el Acoso Escolar .................... 37

Protocolo de actuación y programa de intervención......................................... 39

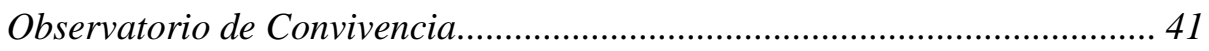

Convivencia Escolar y Aprendizaje ….......................................................... 43 
Actividades de la Asignatura de Intervención y Evaluación en

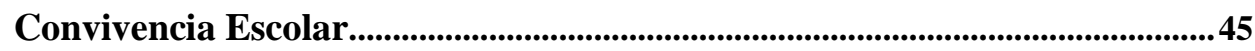

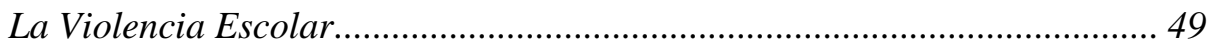

Factores de riesgo que influyen en la conducta del acoso escolar................. 51

Caso práctico: Resolución de conflictos........................................................... 53

Creencias y estereotipos sobre la violencia escolar ...................................... 57

Caso práctico: Resolución de problemas ........................................................ 59

Instrumentos de evaluación de la Convivencia Escolar .................................. 61

Habilidades Sociales y de Comunicación ....................................................... 63

Resolución Pacífica de Conflictos .............................................................. 65

Recursos educativos para la mejora de la Convivencia Escolar................... 67

Actividades de la Asignatura de Investigación en Educación.............................69

Herramientas informáticas en educación ........................................................ 73

Recursos Educativos Digitales.................................................................. 75

El método experimental y no experimental ................................................... 77

Niños con Altas capacidades ........................................................................ 79

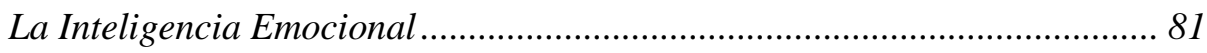

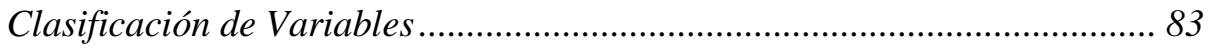

Diseño, muestra y análisis en la investigación cualitativa ............................. 85 


\section{INTRODUCCIÓN}

En la actualidad, la convivencia escolar se convierte en un aspecto fundamental de las relaciones sociales (Caballero, 2010). La preocupación que surge hacia la convivencia escolar son los incipientes problemas de convivencia que se dan en nuestra sociedad, entre los que se encuentra el acoso escolar (Penalva, 2018).

La convivencia escolar está relacionada con el aprendizaje y el bienestar del alumnado, además, está vinculada al desarrollo emocional, social y moral (Gómez-Ortiz, Romera, Ortega-Ruiz, 2017). La convivencia escolar hace referencia a los vínculos que se establecen entre los individuos de la comunidad educativa, siendo un indicador clave para evaluar la calidad educativa (Córdoba, Del Rey, Casas y Ortega-Ruiz, 2016).

La calidad de la convivencia escolar depende de diversos elementos, como pueden ser la calidad de las relaciones interpersonales, el apoyo de los compañeros, la colaboración, la implicación de los estudiantes, así como el desarrollo personal y social y la educación en valores (García, Ortega, y Sola, 2018).

Por todo ello, se plantea la necesidad de desarrollar, elaborar, y valorar planes y programas educativos, destinados a la formación de los docentes, el alumnado, la familia y las comunidades educativas (Sánchez, 2018), con el objetivo de incrementar la mejora de la convivencia escolar, ayudando a que se reduzcan los problemas surgidos en la comunidad educativa y solucionándolos de manera pacífica y fomentando la comunicación y la no violencia en la resolución de conflictos (Sánchez y Sánchez, 2012).

De este modo, el presente manual pretende habilitar al alumnado para la búsqueda y selección de fuentes documentales de carácter científico y dotar al alumno de conocimiento para la recolección y el manejo de los datos, así como completar el Manual de prácticas de los alumnos, y corroborar el aprendizaje adquirido, así como, poner en práctica los conocimientos teóricos adquiridos con el máster.

Para ello, a lo largo del presente manual se desarrollarán una serie de actividades destinadas a alcanzar los objetivos propuestos anteriormente. 
Dichas actividades están organizadas en diferentes materias entre las que encontramos Modelos Teóricos y Bases Conceptuales en Convivencia Escolar, destinada a que el alumnado conozca el concepto Convivencia Escolar, y comprenda como la disrupción en el aula, el acoso escolar, y el rechazo a la población inmigrante son problemáticas comunes a los que los docentes deben dar respuesta cada día.

En relación a Legislación Vigente en Educación y Convivencia Escolar, cuya finalidad es conocer la normativa y las leyes básicas que rigen en las escuelas públicas españolas. Cada actuación relacionada con la convivencia escolar como es la disciplina, el acoso escolar, maltrato infantil o violencia están reglados por decretos y normativas que los futuros docentes deben conocer y que es el marco de referencia para una adecuada función docente.

Por otro lado, se encuentra Intervención y Evaluación en Convivencia Escolar, destinada a que los futuros docentes e investigadores desarrollen y conozcan diferentes instrumentos, necesarios para localizar situaciones que influyan de manera negativa en el clima escolar. Así mismo, es importante conocer qué problemas existen en el aula y qué medidas tomar para solucionarlos y prevenirlos. Por ello, es importante la intervención como la evaluación de situaciones conflictivas en el aula.

Por último, Investigación en Educación, esta materia está destinada a que los futuros docentes dominen y manejen de manera adecuada las nuevas tecnologías, de manera que sea una herramienta que aumente la productividad y facilite la vida diaria de los docentes. De manera que, las actividades desarrolladas se destinen a dominar el análisis y diseño de investigaciones en el ámbito educativo. 


\section{Actividades de la Asignatura de Modelos Teóricos y Bases Conceptuales en Convivencia Escolar}

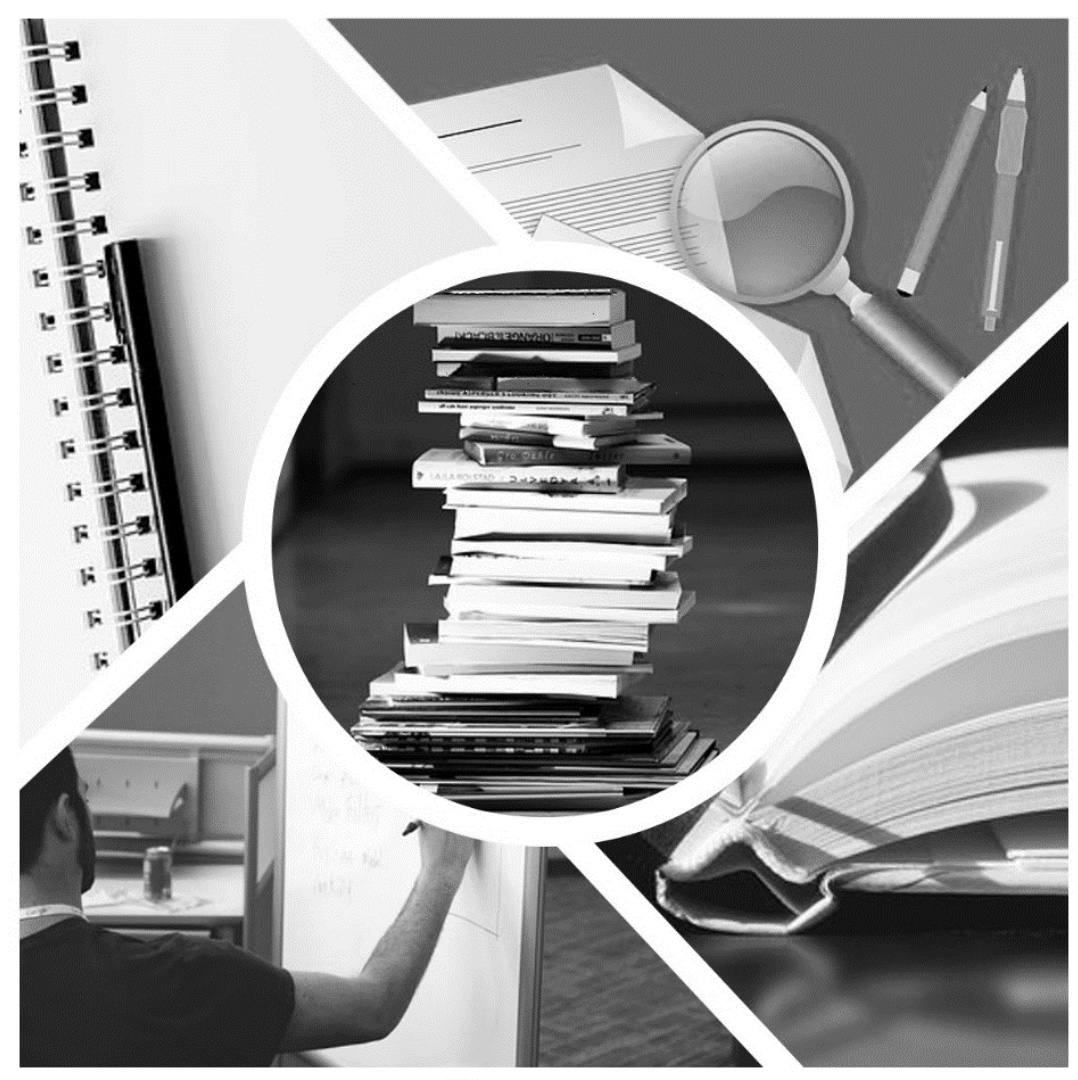



A continuación, se desarrollarán una serie de actividades relacionadas con la asignatura de Modelos Teóricos y Bases Conceptuales en Convivencia Escolar, el objetivo que se pretende alcanzar con las mismas es que el alumnado conozca los modelos teóricos y las bases conceptuales que se desarrollan alrededor de la convivencia escolar, así como, dotar al alumnado de los conocimientos necesarios para la aplicación práctica de diferentes modelos de evaluación e investigación y para la implementación de los instrumentos de recogida de datos cualitativos y cuantitativos de evaluación en educación y en convivencia escolar, además de que adquieran las competencias necesarias para la interpretación de los resultados y la promoción de procesos de intervención a partir de ellos. 



\section{La Convivencia Escolar en el siglo XX}

En primer lugar, descarga el documento "Convivencia y disciplina en el espacio escolar" en el siguiente enlace:

http://www.ub.edu/geocrit/coloquio2014/Jose\%20Antonio\%20Pineda\%20Alfon so.pdf

Tras su lectura y de acuerdo a lo establecido en la misma:

"A mediados de la década de los 90 asistimos en España a una creciente preocupación por una problemática que venía determinada por la percepción, real o imaginada, del deterioro de la convivencia en la escuela. A consecuencia de ello se da una proliferación de informes de expertos, webs especializadas en "violencia escolar", monográficos de revistas educativas y proyectos de investigación dedicados a este tema".

A través de internet, realiza una relación donde indiques y resumas, al menos una de cada tipo de iniciativa (informes de expertos, webs especializadas en "violencia escolar", monográficos de revistas educativas y proyectos de investigación) derivadas de la preocupación de finales de siglo por la convivencia en nuestro país. 
Modelos Teóricos y Bases Conceptuales en Convivencia Escolar

Notas: 


\section{Comienza a conocer la Convivencia Escolar}

En esta actividad en primer lugar deberás acceder a una base de datos (UAL > Biblioteca > Bases de datos > Categorías Educación y/o Psicología) y buscar información sobre publicaciones que traten el tema de la Convivencia Escolar, éstas deben ser anteriores a 2005.

Una vez hayas seleccionado los artículos (debe ser un mínimo de tres artículos), busca la definición que hacen diferentes autores de "Convivencia escolar". Junto a cada una de las definiciones, es importante, que indiques la referencia completa de la publicación a la que corresponde.

Por último, debes realizar una lectura y análisis de todas las definiciones que aportas, identificando los puntos en común y los aspectos nuevos que aporta en comparación al resto. Todo ello, ha de quedar reflejado en un comentario/reflexión sobre este análisis. 
Modelos Teóricos y Bases Conceptuales en Convivencia Escolar

Notas: 


\section{La Convivencia Escolar en la actualidad}

Para comenzar esta actividad primero deberás acceder a una base de datos (UAL > Biblioteca > Bases de datos > Categorías Educación y/o Psicología) y buscar información sobre publicaciones que traten el tema de la Convivencia Escolar actualizados, al contrario que la actividad anterior, deben ser posteriores a 2005.

En los artículos seleccionados (debe ser un mínimo de tres artículos), busca la definición que hacen diferentes autores de "Convivencia escolar". Junto a cada una de las definiciones, es importante, que indiques la referencia completa de la publicación a la que corresponde.

Por último, debes realizar una lectura y análisis de todas las definiciones que aportas, identificando los puntos en común y los aspectos nuevos que aporta en comparación al resto. Todo ello, ha de quedar reflejado en un comentario/reflexión sobre este análisis. 
Modelos Teóricos y Bases Conceptuales en Convivencia Escolar

Notas: 


\section{Comparación crítica y reflexiva}

Realiza una reflexión comparativa y crítica acerca de las diferencias encontradas entre las definiciones de "Convivencia Escolar" anteriores a 2005 (basándote en la Tarea 2a) y posteriores a esta fecha (siguiendo la Tarea 2b).

- ¿Existe alguna evolución en la definición del concepto a lo largo del tiempo?

- ¿Qué líneas comunes plantean?

- ¿Qué diferencias encuentras entre ellas?

Tras la reflexión, realiza una línea del tiempo donde indique la fecha, autores y aspectos más destacados de cada definición. 
Modelos Teóricos y Bases Conceptuales en Convivencia Escolar

Notas: 


\section{Variables y dimensiones de la convivencia escolar}

En el artículo de Del Rey, Ortega, y Feria (2009), se establecieron una serie de variables y dimensiones para la evaluación de la convivencia escolar en el centro, las cuáles se pueden visualizar en la Tabla 2, titulada "Variables y dimensiones de estudio". Puede consultarla en el siguiente enlace:

https://www.aufop.com/aufop/uploaded_files/articulos/1258588302.pdf

A partir de estas, responde a las siguientes preguntas: ¿Crees que son adecuadas y suficientes? ¿Cuál añadirías o quitarías?

Por último, accede a una base de datos (UAL > Biblioteca > Bases de datos > Categorías Educación y/o Psicología) y busca publicaciones donde se evalúe la convivencia en el aula, e indica qué variables y/o factores tienen en cuenta. 
Modelos Teóricos y Bases Conceptuales en Convivencia Escolar

Notas: 


\section{Teorías fundamentales sobre el origen de la agresión}

A continuación, se expone una figura resumen acerca de las teorías fundamentales sobre el origen de la agresión:

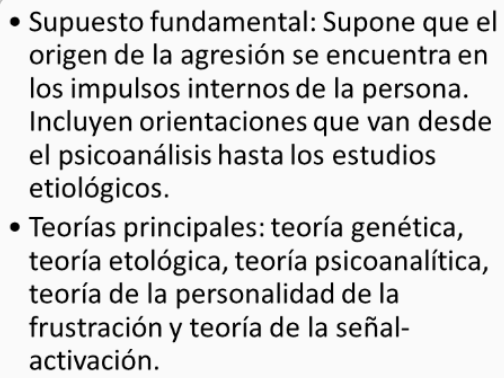

- Teorías principales: teoría genética, teoría etológica, teoría psicoanalítica, teoría de la personalidad de la frustración y teoría de la señalactivación.

- Supuesto fundamental: Suponen que el origen de la agresión se encuentra en el medio ambiente que rodea a la persona, de modo que la agresión en una reacción de emergencia frente a los sucesos ambientales o a la sociedad en su conjunto.

- Teorías principales: Teoría del aprendizaje social, teoría de la interacción social, teoría sociológica y teoría ecológica.

Teorías Reactivas o Ambientales

A través de la consulta en distintas fuentes documentales (bases de datos, biblioteca, páginas web...) debes realizar un cuadro resumen donde indiques la siguiente información acerca de cada una de las teorías innatistas y ambientales: autor, año y breve resumen (entre 5 y 10 líneas). 
Modelos Teóricos y Bases Conceptuales en Convivencia Escolar

Notas: 


\section{Programa de Intervención}

La siguiente actividad se realizará de forma individual. Se diseñará un programa con las siguientes características:

1. Se elegirá una situación conflictiva (inventada) sobre la que realizar el programa de intervención. Esta situación deberá reflejar la relación entre las dificultades de Integración en el Aula y los Conflictos escolares.

2. Se elegirá una edad evolutiva dentro de las etapas de escolarización, infantil, primaria, secundaria o bachiller.

3. Se diseñará un programa que contemple las siguientes partes:

- Nombre del programa. (Alusivo a su contenido).

- Objetivo general. Qué se pretende con el programa.

4. Destinatarios. Descripción psicológica de la edad, nivel y ciclo educativo al que va dirigido dicho programa. Es decir, características evolutivas de los niños (físicas, afectivas, sociales, etc.) a los que va dirigido el programa.

5. Estructura. Esquema del conjunto de unidades que forman el programa. Cada unidad con su nombre correspondiente. Ej. Unidad 1: Me presento, Unidad 2: Preséntate tú, etc.

6. Temporalización. Tiempo óptimo de implantación del programa en meses o cursos.

7. Metodología. Cómo se va a llevar a cabo el programa.

8. Unidades (Se presentarán un total de 4 unidades). Cada unidad que forma la estructura del programa deberá contener la siguiente información:

1. Breve descripción del contenido a trabajar.

2. 1 o 2 objetivos específicos a conseguir en cada unidad.

3. Tiempo (minutos) y lugar para realizar la unidad.

4. Material o recursos que se van a utilizar.

5. Descripción de las actividades para conseguir los objetivos. 
Modelos Teóricos y Bases Conceptuales en Convivencia Escolar

Notas: 


\section{La resolución de conflictos en el aula}

A través de internet, realiza una búsqueda de dos programas a nivel autonómico para la actuación en la resolución de conflictos en el aula. Dichos programas no deben ser andaluces.

Finalmente, basándote en los programas hallados en la búsqueda y en el consultado a través de la Lectura 6 (Programa de Alumno Ayudante, Consejería de Educación de la Junta de Andalucía Dirección General de Participación y Solidaridad en la Educación), realiza una tabla resumen donde indiques nombre, comunidad, características comunes y características diferenciadoras de los programas. 
Modelos Teóricos y Bases Conceptuales en Convivencia Escolar

Notas: 


\section{Actividades de la Asignatura de Legislación Vigente en Educación y Convivencia Escolar}

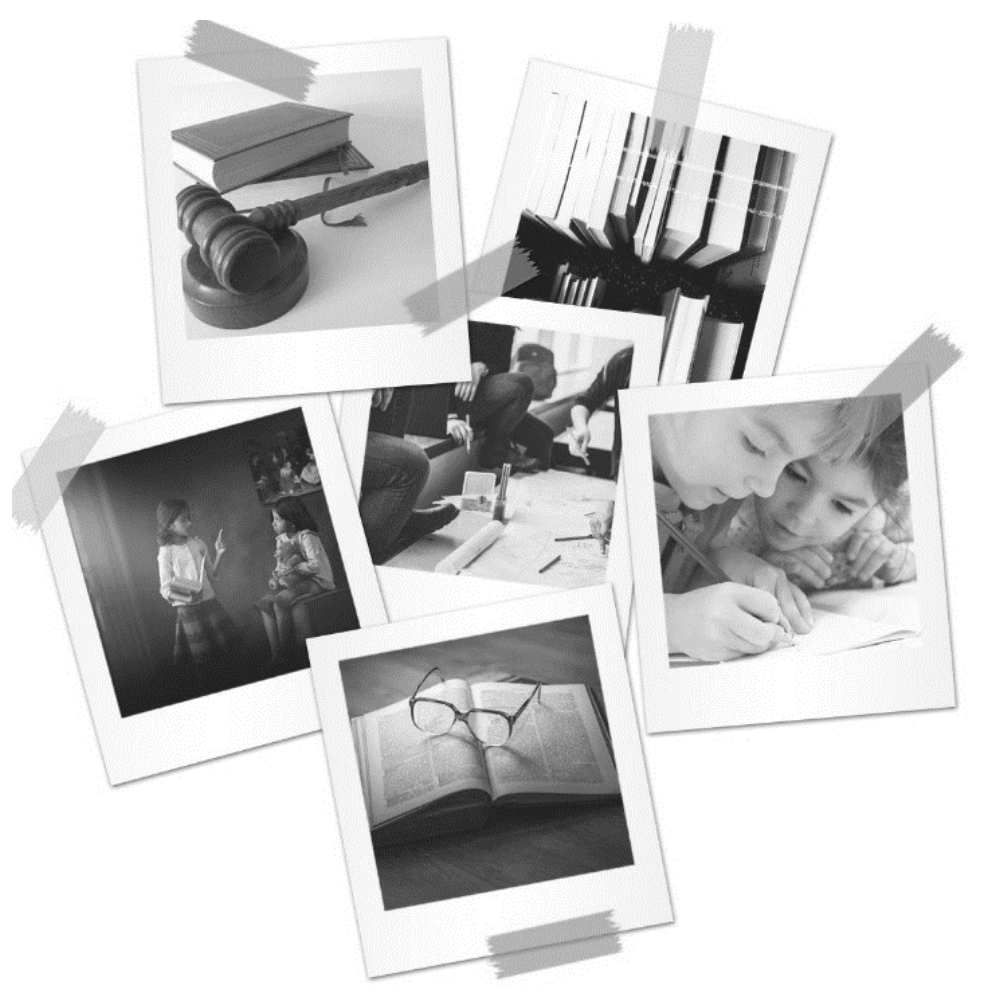



La materia relacionada con Legislación Vigente en Educación y Convivencia Escolar, estará destinada a que el alumnado conozca y comprenda la normativa que conlleva la Convivencia Escolar, así como protocolos de actuación y programas de intervención destinados a solventar los problemas relacionados con la violencia escolar y el acoso escolar. 



\section{El origen de la educación en España y la evolución de las leyes de educación}

Realiza esquema-resumen donde se muestre el origen de la educación en España y la evolución de las leyes de educación. Es decir, un recorrido histórico del sistema educativo español, identificando desde la primera ley que se estableció hasta la que tenemos actualmente. Por otro lado, debes destacar los cambios más significativos que se han producido en esta trayectoria.

Finalmente, debes realizar un breve comentario/reflexión (no más de 300 palabras) acerca de los cambios que ha sufrido la educación a lo largo de los años. 
Legislación Vigente en Educación y Convivencia Escolar

Notas: 


\section{Programas y organismos en Convivencia Escolar}

Realiza una búsqueda de programas y organismos en Convivencia Escolar que existen en Europa y en América Latina. Tras la búsqueda, debes realizar una tabla comparativa entre ambos señalando las diferencias, ventajas, limitaciones, población a la que va dirigida, objetivos, el país donde se creó y si se ha implantado en otros países distintos. 
Legislación Vigente en Educación y Convivencia Escolar

Notas: 


\section{Recursos acerca de la Convivencia Escolar y el Acoso Escolar}

Accede a la página del Ministerio de Educación, Cultura y Deporte sobre Convivencia Escolar:

https://www.mecd.gob.es/educacion/mc/convivencia-escolar/recursos.html

Busca información en el apartado de "recursos" sobre publicaciones que traten el tema de la convivencia escolar y el acoso escolar.

En los artículos seleccionados (debe ser un mínimo de dos artículos) busca las pautas de actuación que se llevan a cabo. Identifica y describe brevemente las más relevantes. Junto a cada actuación, es importante, que indiques la población a la que va dirigida y que incluyas la referencia completa de la publicación a la que corresponde.

Finalmente, debes realizar una lectura y análisis de las actuaciones que has descrito, identificando los puntos en común y destacando los aspectos novedosos que consideras que aporta, en comparación al resto. 
Legislación Vigente en Educación y Convivencia Escolar

Notas: 


\section{Protocolo de actuación y programa de intervención}

Describe un caso sobre violencia escolar inventado, donde tendrás que poner en práctica un protocolo de actuación y desarrollar una intervención para este contexto.

1) Describir el caso, detallando las causas y efectos para poder delimitar esta situación.

2) Identificar el tipo de acoso y las consecuencias sufridas del mismo.

3) Describir el protocolo de actuación paso por paso que llevarías a cabo ante esta situación (teniendo en cuenta la lectura 3 ).

4) Señalar los siguientes apartados de la intervención que realizarías:

a. Dónde, quién y cuándo llevarías a cabo el programa de intervención.

b. Estrategias que emplearías.

c. Evaluación de la intervención. 
Legislación Vigente en Educación y Convivencia Escolar

Notas: 


\section{Observatorio de Convivencia}

1. Contesta la siguiente pregunta:

¿Sabéis lo que es un Observatorio de Convivencia?

2. Haz clic en el siguiente enlace y descarga el documento:

https://dialnet.unirioja.es/servlet/articulo?codigo $=3915955$

3. Tras la lectura del documento anterior "La normativa sobre la Convivencia Escolar en España", realiza un cuadro resumen sobre todo lo concerniente a los Observatorios de Convivencia respecto a la Comunidad Autónoma de Andalucía, así como a la normativa que le afecta a nuestra C.A. 
Legislación Vigente en Educación y Convivencia Escolar

Notas: 


\section{Convivencia Escolar y Aprendizaje}

La convivencia escolar, alude, fundamentalmente, a uno de los temas básicos de la pedagogía: el aprendizaje, es decir, el proceso por el cual un sujeto adquiere o desarrolla una nueva conciencia $y$ conocimiento, que le proporcionan muevos significados.

A partir de la idea anterior, surgen algunas preguntas a las que debes responder:

1. ¿Es posible un adecuado aprendizaje sin una adecuada convivencia?

2. ¿Se puede lograr una buena convivencia sin aprendizaje?

3. ¿Qué significa aprendizaje de la convivencia?

4. ¿Qué función, qué lugar le corresponde a la escuela en relación a convivencia y aprendizaje? 
Legislación Vigente en Educación y Convivencia Escolar

Notas: 


\section{Actividades de la Asignatura de Intervención y Evaluación en Convivencia Escolar}

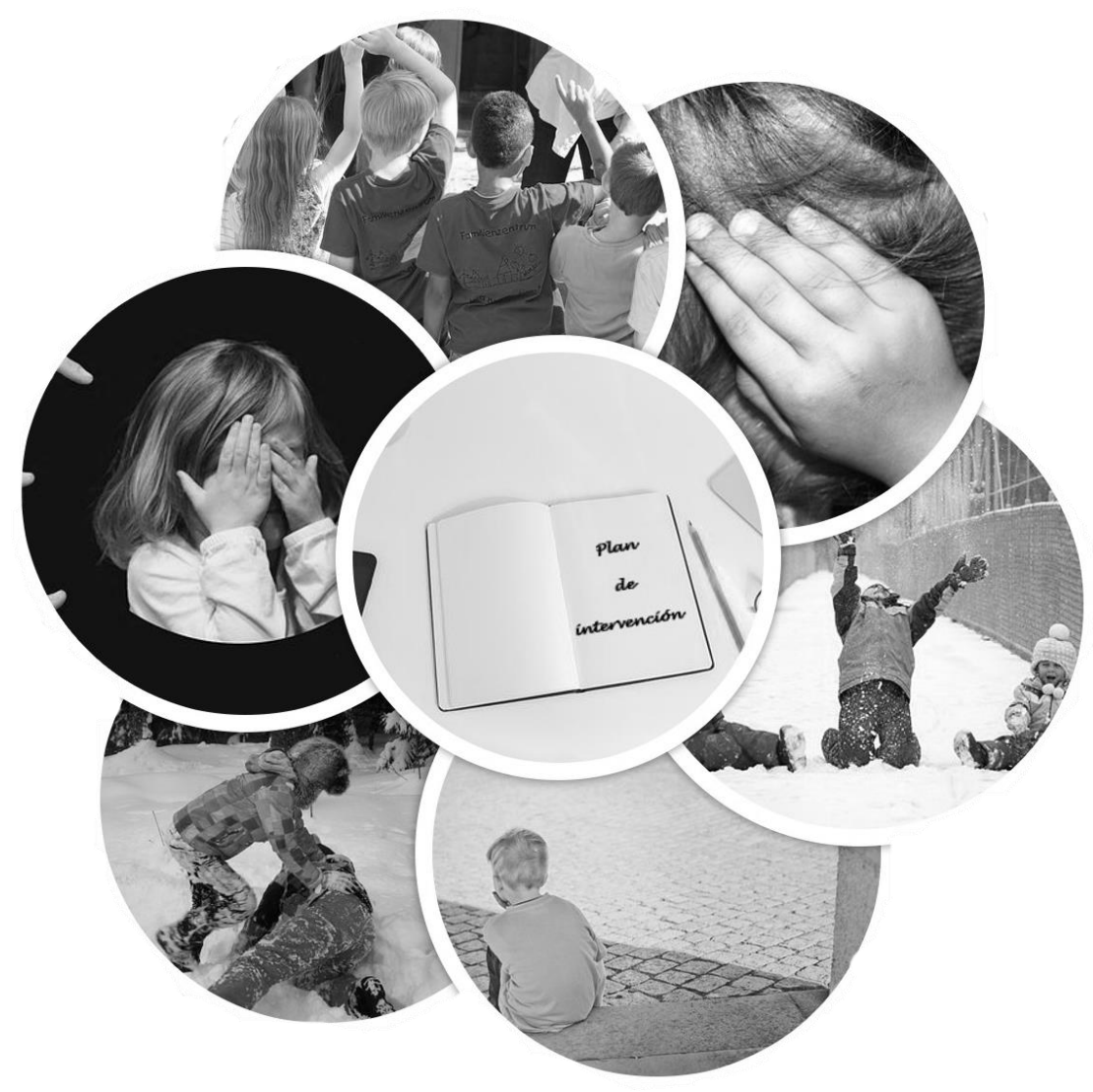



En cuanto a la asignatura de Intervención y Evaluación en Convivencia Escolar, se desarrollarán actividades con la finalidad de que el alumnado sea capaz de conocer Programas de intervención en convivencia escolar, dirigida a la comunidad educativa. Así como, capacitar al alumnado para la identificación de problemas de convivencia y para la evaluación del clima escolar y de los diversos factores que influyen en la convivencia. 



\section{La Violencia Escolar}

En esta primera tarea, deberás acceder a una base de datos (UAL > Biblioteca $>$ Bases de datos > Categorías Educación y/o Psicología) y buscar información sobre publicaciones que traten o donde se identifique algún factor considerado "protector" de las situaciones de violencia escolar.

Una vez que hayas seleccionado los artículos (debe ser un mínimo de dos artículos), describe brevemente en qué consiste cada una de las propuestas y/o conclusiones a las que llegan los autores.

Es importante que, junto a la descripción, indiques la referencia completa de la publicación a la que corresponde. 
Intervención y Evaluación en Convivencia Escolar

Notas: 


\section{Factores de riesgo que influyen en la conducta del acoso escolar}

Se muestra una secuencia de tareas que debes resolver:

1) Acoso escolar y factores de riesgo:

A partir de los resultados presentados en la literatura científica, se habla de algunos factores que parecen influir sobre la conducta de acoso entre iguales. Estos factores se pueden identificar atendiendo a: características individuales, al ambiente familiar, y al medio escolar y social. Pon dos ejemplos de factores de riesgo para cada una de las categorías mencionadas.

2) Factores de riesgo y actuación educativa:

Aunque existen factores de riesgo que inciden en el desarrollo del acoso escolar y sobre los que no se puede actuar directamente desde el centro educativo, ¿crees que es posible desarrollar actuaciones escolares que permitan reducir el impacto de esos factores? Pon un ejemplo.

3) Actuaciones para reducir la influencia de los factores de riesgo:

A tal efecto se plantean medidas para: a) desarrollar las interacciones entre los alumnos; b) establecer modelos adecuados de relación; c) incrementar la vigilancia en el centro; d) desarrollar comportamientos prosociales.

Por último, accede a una base de datos (UAL > Biblioteca > Bases de datos $>$ Categorías Educación y/o Psicología) y busca información sobre alguna de estas medidas para reducir la influencia de los factores de riesgo.

Una vez seleccionada, describe brevemente en qué consiste. 
Intervención y Evaluación en Convivencia Escolar

Notas: 


\section{Caso práctico: Resolución de conflictos}

A continuación, se exponen dos situaciones cotidianas que pueden darse en el aula ordinaria, lee ambas y contesta las preguntas que se plantean a continuación:

\section{Situación A:}

Un día cualquiera en clase:

La clase está aparentemente tranquila pero el profesor observa que A tiene cara de pocos amigos y está mirando a $\mathbf{B}$ de forma no muy amigable. $\mathbf{B}$ le mira y no entiende muy bien lo que está ocurriendo, pero se siente incómodo, parece que A tiene algo contra él. $\mathbf{B}$ decide hablar con $\mathbf{A}$.

B: “¿Estás enfadado conmigo? ¿Por qué?”.

A: "Eres un chismoso, te metes donde no te llaman". El tono de la conversación se va elevando.

B: "No sé qué me estás diciendo, ¿me lo puedes explicar?".

A: ¡Qué! ¿Despistando? ¡Chivato!

B: Que no sé a lo que te refieres, yo no he dicho nada a nadie.

A: (Hace un gesto despectivo). ¡Vete a paseo imbécil!

Después de este diálogo pasan a la acción: A le da un empujón a $\mathbf{B} .$. 


\section{Situación B:}

Ha desaparecido un teléfono móvil. Pedro cree que ha sido Isabel quien se lo ha cogido porque, desde hace unos días, tiene uno igual que el suyo. La profesora le pregunta si ha sido ella la que ha cogido el móvil de Pedro. Isabel le dice que no entiende por qué le hace esa pregunta y explica que es el móvil nuevo que le han comprado sus padres. Conforme avanza la conversación, Isabel se muestra cada vez más nerviosa y termina enfadada, gritando e insultado, y amenazando al compañero. Isabel vuelve a su sitio, abre su cartera y saca su contenido haciendo ruido y hablando por lo bajo.

\section{Tarea:}

Ante una señal de alarma de conflicto en el aula, como las que se presentan en estas situaciones, ¿cuál sería tu respuesta inmediata como docente? (da una respuesta para cada caso). Y, ¿cómo gestionarías esta situación entre los alumnos a largo plazo, en el caso de que se diera de forma repetida en el tiempo? (da una respuesta para cada caso). 
Notas: 



\section{Creencias y estereotipos sobre la violencia escolar}

A continuación, se exponen una serie de cuestiones a las que debes dar respuesta:

1) ¿Cuáles son las creencias y estereotipos sobre la violencia escolar?

2) ¿Qué tipo de debate gira alrededor de las mismas?

3) ¿Cómo son tratadas estas situaciones en el ambiente escolar, familiar o en los medios de comunicación?

4) En algunos de los estudios realizados durante la última década, se incide junto a la violencia entre alumnos, la violencia hacia los profesores, y se señalan una serie de mitos, entre los que destacan:

A. La violencia escolar no ha aumentado en los centros. Desde esta perspectiva se sostiene que es la de siempre, que siempre ha existido, sólo que ahora hay más sensibilidad social.

B. La violencia escolar es una novedad. Este planteamiento basa su justificación en los "tiempos que vivimos", en la falta de autoridad, en la falta de respeto a los demás, en situaciones especiales.

C. La violencia escolar se da sólo de forma aislada y en situaciones muy específicas. Desde este punto de vista, no debe generalizarse y crear alarma social ¿Qué hacer? ¿Qué postura adoptar?

Comenta brevemente cada uno de los mitos expuestos, ¿estás de acuerdo? Y ¿qué consecuencias pueden tener sobre nuestra forma de actuar ante situaciones de violencia escolar?

5) Accede a una base de datos (UAL > Biblioteca > Bases de datos > Categorías Educación y/o Psicología) y busca algún artículo de revista en el que se aporten datos que contradigan alguna de las afirmaciones de los mitos expuestos en el punto 4. Solamente tienes que escribir aquí la referencia del artículo seleccionado. 
Intervención y Evaluación en Convivencia Escolar

Notas: 


\section{Caso práctico: Resolución de problemas}

Siguiendo el ejemplo que se presenta a continuación, plantea un problema de indisciplina en el aula, y sigue los pasos para su resolución.

\section{Paso 1: ¿Qué? ¿Por qué?}

1.1. Definir el problema:

Elvira tira la leche todos los días en el desayuno escolar.

1.2. Identificar situaciones en las que aparece/n y antecedentes:

- Todas las mañanas.

- Siempre que bebe leche.

- La madre dice que no le gusta la leche.

1.3. Análisis causas posibles: proponer y razonar alternativas:

- No le gusta la leche, quiere evitar beber/ puede ser porque pone mala cara....

- No sabe beber en tazal no pues ha bebido agua en alguna ocasión.

- Quiere atraer mi atención.

1.4. Seleccionar una propuesta:

No le gusta la leche, la quiere evitar.

\section{Paso 2: Cómo prevenir}

2.1. Redefinir el problema, si es posible, en términos positivos:

- ¿Cómo hacer que Ana tome un desayuno saludable?

- ¿Cómo hacer para que Ana no tire la leche aunque no la quiera? 
2.2. Razonamiento de alternativas y consecuencias.

- Darle otros alimentos equivalentes.

- Darle leche con cacao.

- Enseñarle a decir "no quiero".

\section{Paso 3: Cómo actuar}

1.1. Definir el problema en términos más-menos:

El problema es que tira la leche porque no se la quiere tomar.

1.2. Razonamiento de alternativas y consecuencias.

- Mandarla la silla de pensarl ¿?

- Hacer que lo limpiel ¿?

- Regañarlal ¿?

3.3. Puesta en práctica y valorar resultados

\section{Notas:}




\section{Instrumentos de evaluación de la Convivencia Escolar}

Se llevará a cabo un análisis de los instrumentos disponibles para evaluar diferentes aspectos relacionados con la convivencia escolar.

1. Se debe seleccionar un instrumento de evaluación, de manera que se confeccione un listado con diferentes propuestas.

2. Se trata de que cada alumno realice una búsqueda de información (ventajas, inconvenientes, población a la que se dirige, si es accesible, si ha sido validado en nuestro país, su utilidad en la práctica docente o en el diseño de programas de intervención, etc.) sobre todos los instrumentos propuestos en la actividad anterior.

La información recopilada por el alumno, sobre cada instrumento, será presentada en una ficha que recoja toda la información, cuyo modelo se mostrará a continuación.

\section{NOMBRE DEL INSTRUMENTO}

CARACTERÍSTICAS

\begin{tabular}{|l|}
\hline Objetivos \\
\hline$N^{\mathbf{o}}$ de ítems: \\
\hline Dimensiones: \\
\hline $\begin{array}{l}\text { Escalas } \\
\text { subescalas: }\end{array}$ \\
\hline
\end{tabular}

Población a la que se dirige:

\section{Edades:}

Palabras clave

(entre 3 y 5):

Otros datos de

interés:

Autor/a:

Referencias:

Enlace a la

publicación: 
Intervención y Evaluación en Convivencia Escolar

Notas: 


\section{Habilidades Sociales y de Comunicación}

Para realizar la presente tarea deberás contestar las cuestiones que aparecen a continuación:

1) ¿Qué entiendes por "Habilidades Sociales y de Comunicación”?

2) ¿Cuál crees que puede ser la utilidad de su evaluación, en relación a los problemas de convivencia escolar?

3) Según aquello que entiendo que son y para lo que sirven estas habilidades, ¿cuáles de ellas consideras que son más importantes trabajar en el alumnado? ¿Y en el caso del profesorado?

4) Enumera algunas de las dificultades que pueden presentar los instrumentos destinados a evaluar las Habilidades Sociales y de Comunicación. Puedes poner ejemplo/s. 
Intervención y Evaluación en Convivencia Escolar

Notas: 


\section{Resolución Pacífica de Conflictos}

A nivel mundial, se han llevado a cabo diferentes iniciativas para la Resolución Pacífica de Conflictos (Verdiani, 2000). Algunas de ellas se muestran a continuación:

Canadá: La Maison des Jeunes de l'Azymut Est-Ouest de San Nicolás de Québec ha confeccionado una Guía de Formación en Comportamientos Pacíficos. Así como el desarrollo de diversas experiencias de sensibilización y de educación para la paz con alumnos de educación primaria y secundaria a través del programa "Embajadores y embajadoras de la paz" durante 1999. Una de las actividades desarrolladas por el programa es la elaboración de marionetas que simbolicen la paz en un taller dirigido al alumnado de 5 a 7 años, poniendo en práctica una serie de unidades didácticas.

Finlandia: En el programa dirigido por Christina Salmivalli del Departamento de Psicología de Universidad de Turku, han participado 48 cursos de 16 escuelas de Helsinki y Turku a través de una formación específica impartida al profesorado que más tarde ha implementado el programa en sus respectivas clases. La característica de este proyecto se basa en los mecanismos colectivos que intervienen para prevenir el maltrato entre iguales.

India: Gracias a la iniciativa del Centro de Trabajo y de Investigación (Social Work and Research Centre), se han creado 150 escuelas dentro del proyecto "La escuela de los pies desnudos". Este proyecto implantado en la zona norte de la India, a inicios de la década de los 80 , desarrolla diversos programas sociales entre los que se incluye el de resolución no violenta de los conflictos. Dirigido a mejorar las relaciones no sólo del alumnado, sino también del profesorado y de sus familias a través de un conjunto de actividades contenidas en un manual didáctico. 
La tarea consiste en:

1. Buscar información acerca de las iniciativas presentadas (u otras similares), ampliar la información con otros datos y expresar la opinión en cuanto a la idoneidad de la metodología, los recursos utilizados, los resultados obtenidos, etc.

2. Según tú opinión, ¿se obtendrían los mismos resultados si se aplican estos programas en nuestro país? Justificar la respuesta.

3. Buscar un programa para la Resolución Pacífica de Conflictos que haya sido puesto en marcha recientemente en nuestro país y explicar brevemente en qué consiste. 


\section{Recursos educativos para la mejora de la Convivencia Escolar}

1) Realizar una búsqueda de recursos educativos desarrollados a la mejora de la Convivencia Escolar. Para ello, el alumno puede acceder a las webs del Ministerio o de las Comunidades Autónomas.

2) El trabajo consiste en el diseño de materiales educativos orientados a la mejora de la Convivencia Escolar. Deben ser recursos de creación propia, donde se valorará la originalidad, la utilidad para el objetivo planteado, y el grado de ajuste a las características de la población a la que se dirige. No es necesario que sean materializados (cuando se trate de trabajos plásticos y/o artísticos), en ese caso, se considera suficiente con el desarrollo de la propuesta, siempre y cuando esté suficientemente detallada. 
Intervención y Evaluación en Convivencia Escolar

Notas: 


\section{Actividades de la Asignatura de Investigación en Educación}

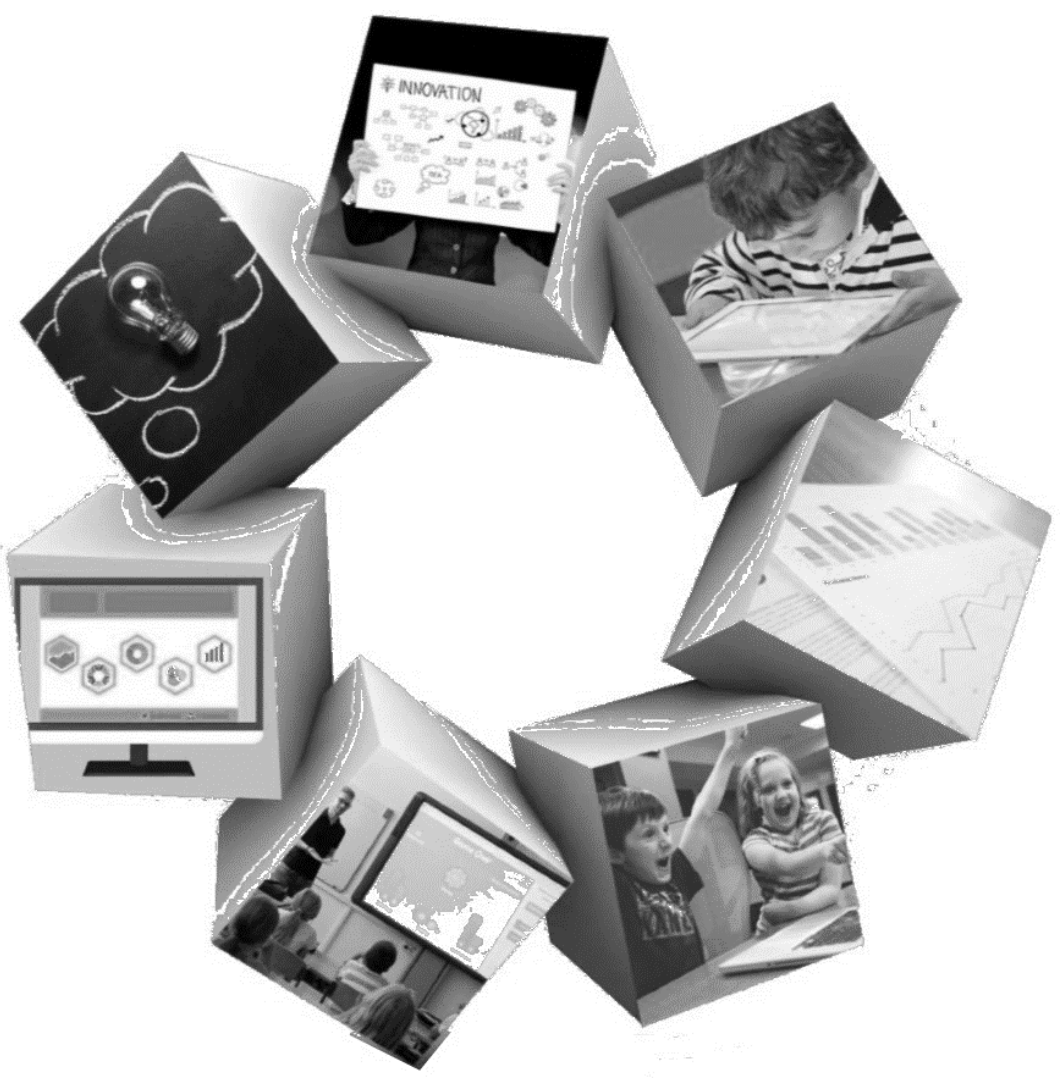



A continuación, se muestran una serie de ejercicios enfocados a trabajar en profundidad la asignatura de Investigación en Educación, de este modo, se procurará que los futuros docentes comprendan y realicen de manera adecuada el análisis y diseño de investigaciones en el ámbito educativo. Análisis de datos. Programas estadísticos informáticos para la investigación en educación cualitativa y cuantitativa. 



\section{Herramientas informáticas en educación}

Accede a una base de datos (UAL > Biblioteca > Bases de datos $>$ Categorías Educación y/o Psicología) y busca información sobre publicaciones que traten el tema de las herramientas informáticas en educación.

En los documentos seleccionados (mínimo 2), busca una herramienta educativa utilizada. Junto a cada una de las herramientas y/o recursos, es importante, que indiques la referencia completa de la publicación o fuente consultada.

Igualmente, debes realizar un breve comentario sobre cada uno de los recursos informáticos que has seleccionado, identificando a quien se dirige (alumnos, docentes, orientadores...) y en qué consiste. Finalmente, señala, según tu opinión, los beneficios que pueden aportar cada herramienta a la comunidad educativa. 
Investigación en Educación

\section{Notas:}




\section{Recursos Educativos Digitales}

En primer lugar, accede a la siguiente dirección de internet:

http://www.educacontic.es/

Seguidamente, pincha sobre la pestaña "Catálogo TIC" (situado en la barra superior de la página). Entre los recursos educativos que aparecen, selecciona uno de ellos y realiza una breve descripción de la entrada (extensión máxima: 1 página). 
Investigación en Educación

Notas: 


\section{El método experimental y no experimental}

A continuación, se exponen algunas de las características del método experimental y no experimental:

\begin{tabular}{|c|c|}
\hline $\begin{array}{l}\text { INVESTIGACIÓN } \\
\text { EXPERIMENTAL }\end{array}$ & $\begin{array}{l}\text { INVESTIGACIÓN NO } \\
\text { EXPERIMENTAL }\end{array}$ \\
\hline $\begin{array}{l}\text { Requiere de una manipulación rigurosa } \\
\text { de las variables, y del control directo o } \\
\text { por procedimientos estadísticos al azar, } \\
\text { de otros factores que pueden afectar el } \\
\text { experimento. } \\
\text { Estos procedimientos al azar incluyen a } \\
\text { selección al azar de los sujetos, la } \\
\text { asignación a azar de los sujetos a los } \\
\text { grupos experimentales y de control y la } \\
\text { asignación al azar del tratamiento } \\
\text { experimental a uno de los grupos. }\end{array}$ & 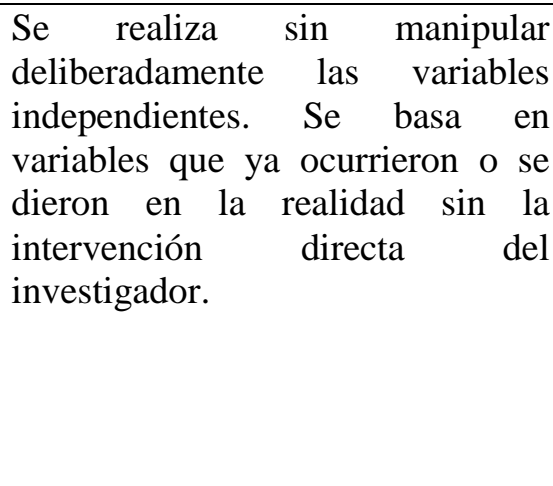 \\
\hline $\begin{array}{l}\text { Emplea un grupo de control para } \\
\text { comparar los resultados obtenidos en el } \\
\text { grupo experimental, teniendo en cuenta } \\
\text { que, para los fines del experimento, } \\
\text { ambos grupos deben ser iguales, excepto } \\
\text { en que uno recibe un tratamiento (el } \\
\text { factor causal) y el otro no. }\end{array}$ & $\begin{array}{l}\text { Se basa en variables que ya } \\
\text { ocurrieron o se dieron en la realidad } \\
\text { sin la intervención directa del } \\
\text { investigador. }\end{array}$ \\
\hline $\begin{array}{l}\text { La investigación experimental es el } \\
\text { procedimiento más adecuado para } \\
\text { investigar relaciones de causa - efecto, } \\
\text { pero es artificial y restrictiva. }\end{array}$ & $\begin{array}{l}\text { Es un enfoque retrospectivo. Es } \\
\text { conocida también como } \\
\text { investigación expostfacto (los } \\
\text { hechos y variables ya ocurrieron) y } \\
\text { observa variables y relaciones entre } \\
\text { estas en su contexto natural. }\end{array}$ \\
\hline $\begin{array}{l}\text { Ejemplo: Hacer enojar intencionalmente } \\
\text { a una persona para ver sus reacciones. }\end{array}$ & $\begin{array}{l}\text { Ejemplo: Ver las reacciones de esa } \\
\text { persona cuando llega enojado. }\end{array}$ \\
\hline
\end{tabular}

En base a las características de la investigación experimental y no experimental expuestas en el cuadro anterior, determina, de forma razonada, qué tipo de investigación es la siguiente:

Un investigador desea analizar el efecto que produce el consumo de alcohol sobre los reflejos humanos en hombres. Para ello, asignará al azar a los sujetos a 
los siguientes grupos: un grupo donde los sujetos ingerirán un elevado consumo de alcohol (7 copas de tequila), un segundo grupo que ingerirán un consumo medio de alcohol (4 copas), un tercer grupo que beberá un consumo bajo de alcohol (una copa) y un cuarto grupo que no ingerirá nada de alcohol. Controlará el lapso en el que todos los sujetos consumirán su "ración" de alcohol, así como otros factores (misma bebida, cantidad de alcohol servida por copa, consumo previo de alimento sólido, etc.). Finalmente, medirá la cantidad de respuesta de los reflejos en cada grupo y comparará los grupos, para así determinar el efecto del consumo de alcohol sobre los reflejos humanos.

\section{Notas:}




\section{Niños con Altas capacidades}

A. Descarga el siguiente documento "Desarrollo del Protocolo de Observación de Interacción en el Aula: aplicación en un programa de niños con altas capacidades" en el siguiente enlace y realiza su lectura:

http://www.mecd.gob.es/dctm/revista-deeducacion/doi/re2013250.pdf?documentId=0901e72b8170bd38

B. Tras dicha lectura, elabora una tabla de registro para evaluar, a través de la observación, el mal comportamiento de un alumno dentro del aula. Recuerda que, primero, debes definir qué conductas vas a observar (número de veces que habla sin levantar la mano, tiempo que está fuera de su sitio...). 
Investigación en Educación

Notas: 


\section{La Inteligencia Emocional}

Desde el portal de la universidad, accede a la biblioteca Nicolás Salmerón. Desde el apartado "bases de datos", accede a Dialnet Plus.

Una vez en esta, realiza una búsqueda acerca de las publicaciones relacionadas con la Inteligencia Emocional y responde a las siguientes preguntas:

1) ¿Qué descriptor o descriptores has utilizado?

2) ¿Utilizaste operadores booleanos? En caso afirmativo, indica cuales.

3) Indica la fórmula de búsqueda completa que has utilizado.

4) ¿Cuáles fueron los filtros qué usaste?

5) ¿Cuántos resultados obtuviste antes de introducir los filtros? ¿Y cuantos después? 
Investigación en Educación

Notas: 


\section{Clasificación de Variables}

Indica si las siguientes variables son escalares, nominales u ordinales:

$>$ Género.

$>$ Número de horas que los niños ven la televisión.

$>$ Estado civil.

$>$ Intensidad del dolor (mucho dolor, dolor medio, poco dolor, sin dolor).

$>$ Medida de talla de un niño (alto, bajo, medio).

$>$ Medida de la talla de un niño (en centímetros). 
Investigación en Educación

Notas: 


\section{Diseño, muestra y análisis en la investigación cualitativa}

Responde a las siguientes preguntas tras realizar la lectura del documento "Diseño, muestra y análisis en la investigación cualitativa". Puedes descargar el documento en el siguiente enlace:

http://www.cienciared.com.ar/ra/usr/3/206/n7_vol3pp123_146.pdf

1. ¿Cómo se denomina al proceso de interacción social que da como resultado la realidad vivencia por cada individuo?

2. ¿Para qué se utilizan las formas ideológicas?

3. ¿Cómo es el diseño de los estudios cualitativos?

4. ¿Qué significa que la estrategia cualitativa de producción de datos es recursiva?

5. ¿En qué dos criterios se basa la selección de los entrevistados en la metodología cualitativa?

Define con tus palabras en qué consiste el análisis de atribución y frecuencia como opción a la hora de elegir el tipo de análisis de datos en la investigación cualitativa. 
Investigación en Educación

Notas: 


\section{Referencias}

Caballero, M.J. (2010). Convivencia escolar. Un estudio sobre buenas prácticas. Revista Paz y Conflictos, (3), 154-169.

Córdoba, F., Del Rey, R., Casas, J.A., y Ortega-Ruiz, R. (2016). Valoración del alumnado de primaria sobre convivencia escolar: El valor de la red de iguales. Psicoperspectivas, 15(2), 79-90.

García, M., Ortega, M.C., y Sola, J.M. (2018). La importancia de los valores transmitidos por el profesorado de secundaria para una mejor convivencia escolar. Teoría de la Educación, 30(2), 201-221. doi: 10.14201/teoredu302201221

Gómez-Ortiz, O., Romera, E.M., y Ortega-Ruiz, R. (2017). La competencia para gestionar las emociones y la vida social, y su relación con el fenómeno del acoso y la convivencia escolar. Revista Interuniversitaria de Formación del Profesorado, 88(31.1), 27-38.

Penalva, A. (2018). La convivencia escolar. Un reto del siglo XXI. Revista Electrónica de Investigación y Docencia (REID), 20, 41-58. doi: 10.17561/reid.n20.3

Sánchez, S. (2018). La convivencia escolar desde la perspectiva de investigación para la cultura de paz. Participación Educativa: Revista del Consejo Escolar del Estado, 5(8), 57-68.

Sánchez, S., y Sánchez, A. (2012). La convivencia escolar desde la perspectiva de la Cultura de Paz. Convives, 36-41. 
Edita: ASUNIVEP 